\title{
Normal and abnormal red blood cell recognition using image processing
}

\author{
Hajara Abdulkarim Aliyu', Mohd Azhar Abdul Razak², Rubita Sudirman ${ }^{3}$ \\ ${ }^{1}$ Faculty of Electrical Engineering, Jigawa State Polytechnic, Nigeria \\ ${ }^{1,2,3}$ School of Electrical Engineering, Universiti Teknologi Malaysia, Johor Bahru, Malaysia
}

\begin{tabular}{l} 
Article Info \\
\hline Article history: \\
Received Jun 27, 2018 \\
Revised Dec 10, 2018 \\
Accepted Jan 17, 2019 \\
\hline
\end{tabular}

\section{Keywords:}

Form factor

Image Processing

Red blood cells (RBCs)

\begin{abstract}
In medical field, the recognition of red blood cells (RBC) is used as an indicator to detect the type of diseases such as anaemia, malaria and leukaemia etc. The problems using manual detection of normal and abnormal RBCs under the microscope is tend to give inaccurate result and errors. This paper proposed a method to recognize the normal and abnormal shaped RBCs image by using Form Factor, Perimeter and Area as feature descriptors. Detecting normal cells of RBCs indicate a healthy patient and abnormal cells indicate presence of disease. And is very important in medical field to detect abnormal condition in early stage because it saves and protects human lives. The patients waiting time for blood test is more because the time taking to generate the result of the patient is high due to high demand and less equipment this method is used in order to improve the accuracy of the existing one and $94 \%$ accuracy was achieved in the detection.
\end{abstract}

Copyright $(2019$ Institute of Advanced Engineering and Science. All rights reserved.

\section{Corresponding Author:}

Rubita Sudirman

School of Electrical Engineering,

Universiti Teknologi Malaysia, 81310 UTM Johor Bahru.

Email: rubita@utm.my

\section{INTRODUCTION}

The human blood consists of three major components of blood cells which are red blood cell (RBC), white blood cell (WBC) and platelets. Red blood cell has the major percentage in the human blood and conducts various functions in human body such as transporting oxygen throughout the body, carrying carbon dioxide and waste products away from cells and tissue. Normal shape of the RBC is biconcave disks with about 7 to $8 \mu \mathrm{m}$ in diameter and $2.2 \mu \mathrm{m}$ thick [1].

Considering the abnormal RBC morphology give a sign of anaemia, reduction of haemoglobin (a protein that bind with oxygen molecule in RBC), and secondary effect of several other disorders. From medical perspective, the RBC diagnosis contributes information about various blood related disease. For instance, the shape of RBCs and its deformability has connection to the relevant disease more especially anaemia and secondary effect of several other disorder [2]. Anaemia and blood related disorders are common in almost $24.5 \%$ of world population. This make all pathological laboratories the current method of blood smear analysis relies on visually inspecting the blood smear slide under the microscope. The method is time consuming, laborious, expensive and it requires skilled technicians [3].

The shape of abnormal RBCs differs greatly depending on the kind of blood disorder the patient is suffering from. The major steps in image analysis are pre-processing, image segmentation, feature extraction and image detection. The most important and challenging step is image segmentation and feature extraction to enable us detect the exact feature of the blood cells. Many researchers have carried out similar research since some decades ago and still the area need more effort to solve erythrocyte problems, Sheikh et al. [5] used neural network to identify abnormality in Red blood cell, White blood cells and platelets in anaemic condition. Abnormal red blood cell was characterised based on parametric deformable template by Brokorsta et al. [6]. 
MLP (Multilayer perception) was used to classify normal RBCs and sickle cells by Poomcokrak [7]. Elsalamony [8] proposed a method to detect healthy and unhealthy RBCs using Hough transform and neural network. They introduced a methodology that employs machine learning techniques for characterizing RBCs in anaemia based on microscopic images of peripheral blood smears and they used 25 set of features for identification but observed that using a small set of feature will present higher accuracy than they achieved. In this paper we propose a computer aided system to automatically detect normal and abnormal red blood cell (RBC) in blood smear image by using Form factor and perimeter as features to detect the fast and effectiveness for emergency purpose. The rest of the paper is organized as follows: Method of research which describes the proposed method followed by result of the study, discussion and conclusion with acknowledgement. Figure 1 shows normal and abnormal RBCs.

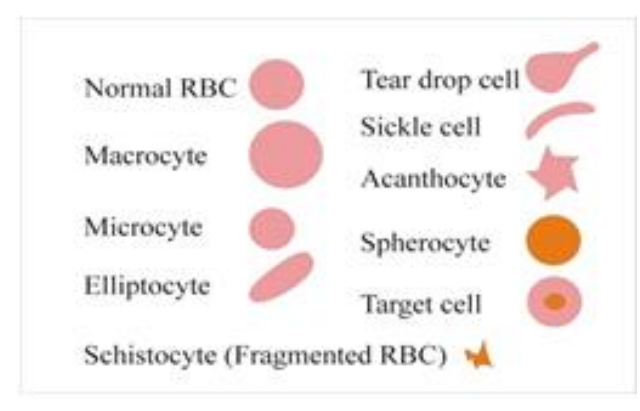

Figure 1. Normal and abnormal rbc's [4]

\section{RESEARCH METHOD}

The method pipeline for automatic detection of the blood cell is shown in Figure 2 which consist of original sample image of normal and abnormal blood cells on a blood smear image, the image is further converted from RGB to Grayscale image, otsu thresholding, image complementing, hole filling, image cropping, feature extraction using form factor and svm model was introduced to classify the normal and abnormal blood cells.

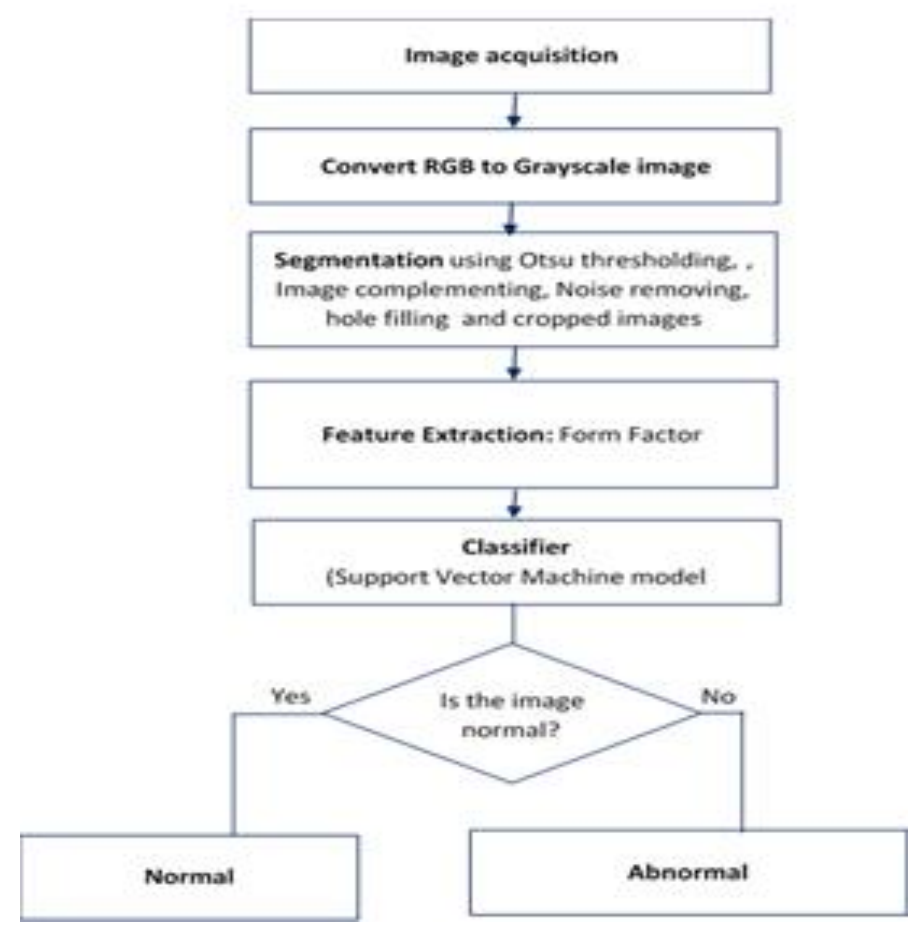

Figure 2. Method pipeline 


\subsection{Image Acquisition}

The blood smear images of the abnormal blood cells were downloaded online from Ash bank and shutter stock while the normal images were collected from the hospital so database is needed for Red blood cell research to be done on human like the dataset that was generated for fingerprints [9]. Figure 3 shows sample of blood images for normal and abnormal smear images. In Figure 3(c), it is clearly seen the shape difference between the normal red blood cells and the sickle cells.

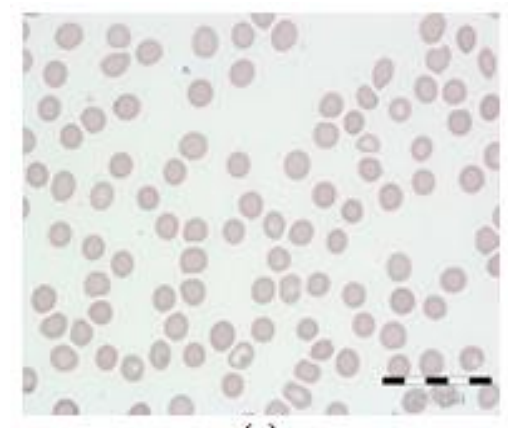

(a)

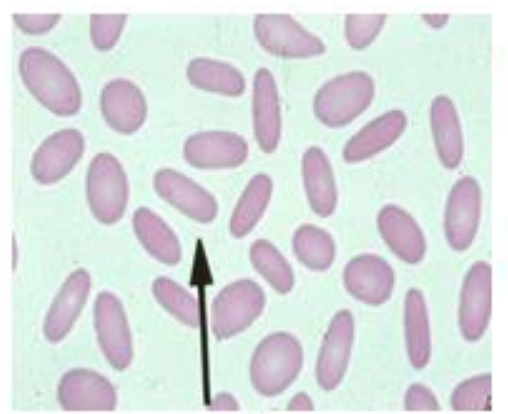

(b)

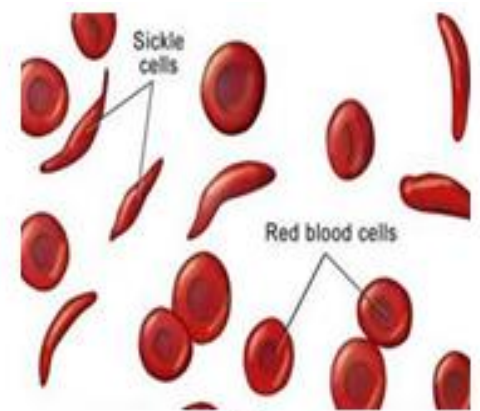

(c)

Figure 3. (a) Normal blood cell images, (b) and (c) The abnormal blood smear images

\subsection{PreProcessing}

The original images are converted to greyscale images which are easier for processing. Hence the input image is converted from colour image to grayscale image. The image that has passed through preprocessing step will be used to decide the segmentation point [10].

\subsection{Segmentation}

Image segmentation is a method that split an image to a group of non-overlapping areas [11]. Thresholding aims at separating RBC's from background and other unwanted objects. Binarization is also technically known as thresholding converts a grayscale image into a binary image which has only two colours black and white. Thresholding act as a segmentation process that is done to ease the job of tracing the boundaries. And Otsu threshold is a global thresholding method which uses a single global threshold to separate the foreground pixels from background pixels. This method is very effective in segmenting the RBCs on blood smear image without losing important information.

\subsection{Image Complementing}

We intend to obtain the RBCs on a darker background so as to get better processing. The image was complemented. The Complemented image is easier for processing and it provides more information than the uncomplemented image.

\subsection{Noise Removal}

The blood smear images consist of unnecessary details like small spots, noise as well as unwanted cells like blood platelets etc. This noise will convey wrong information in RBC counting. Platelet size can be as large as 2500 pixels. Hence any group of pixels smaller than 2500 pixels is considered as noise and is eliminated. The difference between the original images and the images after noise removal can be seen beween Figure 3 and Figure 4. It is a better image representation because the aim of the study is to classify the normal and abnormal cell based on the shape, rather than the color of the image.

\subsection{Cropped Images}

The images are cropped from the blood smear to generate the dataset of normal and abnormal cell in order to train the SVM model for the detection of all kinds of blood cell images. The cropped dataset images are made of 65 normal blood cells and 100 abnormal blood cells. These are illustrated in Figures 5 and 6. 


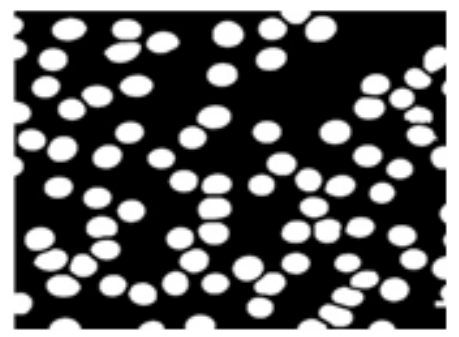

(a)

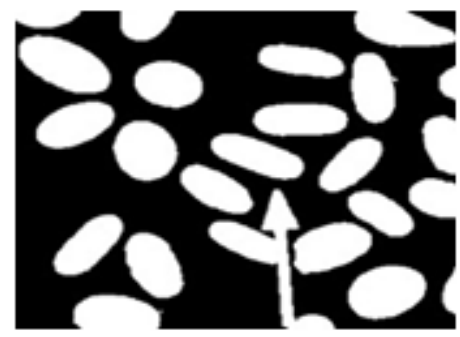

(b)

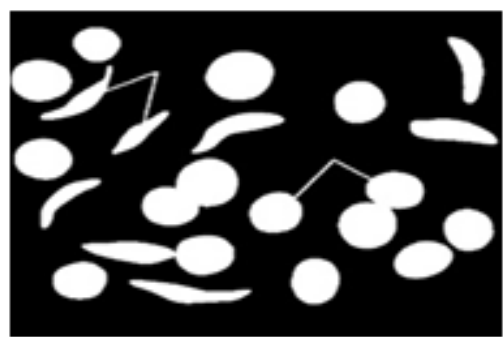

(c)

Figure 4. (a) Normal blood cell images, (b) and (c) The abnormal blood smear images all preprocessed and segmented
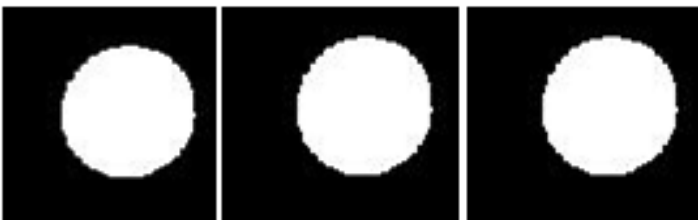

Figure 5. Normal Cropped RBCs Images
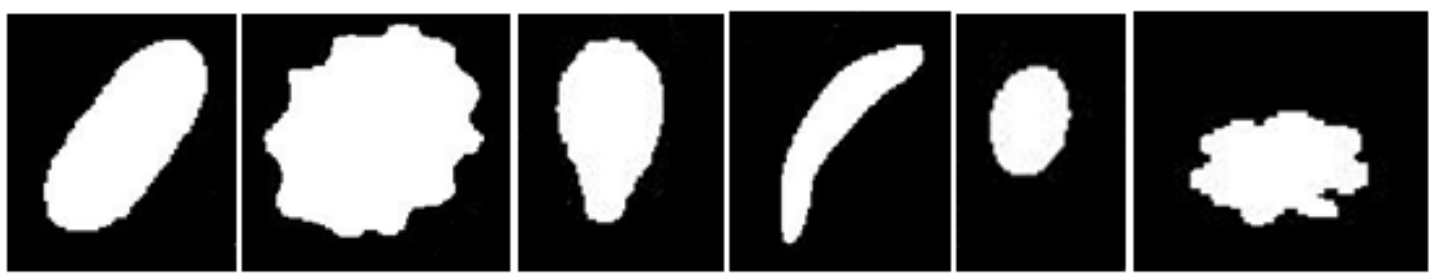

Figure 6. Abnormals Cropped RBCs Images

\subsection{Feature Extraction}

Three features have been identified in classifying the RBCs into normal and abnormal cells types in the work because using small set of features increase the accuracy and reduced the timing of the computation [12]. The details of each feature are explained as follows:

Perimeter - Perimeter is the Sum of pixels around the boundary of the RBC.

Area - Area is the Number of pixels enclosed inside the RBC's boundary

Form Factor - Form factor is calculated for the labelled cell based on area and perimeter as follows:

Form factor $=(4 \times \pi \times$ area $) /$ Perimeter $^{2}$

\section{RESULT AND DISCUSSION}

In this section, the performance of proposed RBC classification system is evaluated for recognizing the $\mathrm{RBC}$ cell type, cropped from the blood smear images in Figure 5. The range of the form factor is in between 0.82 to 1.02 for the normal blood cells and the abnormal blood cells are between 0.8 to 0.3 .

In order to be able to detect between normal and abnormal RBC in the image by using the selected feature, a classifier was used. The classification module is performed by using support vector machine classifier, the value of error function minimized by a one-dimensional linear SVM trained to classify learning samples on the basis of the features of interest. The smaller this error the better is the quality of the feature[13]. The proposed SVM model classifier has been trained tested and validate. Moreover, a best model was achieved by training the normal and abnormal RBCs, which leads to best accuracy. 


\section{CONCLUSION}

The paper proposes an image processing method for detecting normal and abnormal blood cells. The proposed method detects the blood cells with accuracy of $94.12 \%$, specificity of $89.47 \%$ and sensitivity of $100 \%$.this shows that the proposed system is very accurate, cost effective, time saving and is an excellent application of computer vision in aiding blood pathological information. The proposed system will reduce time, cost and labour involved in manual classification of RBCs. The three features proposed are significant and sufficient in RBCs detection. The procedure was done on mat lab 2015a.

\section{ACKNOWLEDGEMENT}

The authors also would like to express their deepest thanks and appreciation to the support by Research Universiti Grant of Universiti Teknologi Malaysia under vot number 14J23.

\section{REFERENCES}

[1] H. A. Aliyu, "Detection of Accurate Segmentation in Blood Cells Count - A Review," Int. J. Sci. Dev. Res., vol. 2, no. 8, pp. 28-32, 2017.

[2] S. C. Webster, J. G. and Cazzanti, Bioinstrumentation-Hematology. John Wiley and Sons, 2004.

[3] Pooja Tukaram Dalvi and Nagaraj Vernekar, "Computer Aided Detection of Abnormal Red Blood Cells," IEEE Int. Conf. Recent Trends Electron. Inf. Commun. Technol., pp. 1741-1746, 2016.

[4] Https://bigpictureeducation.com/blood-cells-images, "No Title." .

[5] M. Sheikh, H., Zhu, B. \& Tzanakou, "Blood cell identification using neural networks.," Twenty-Second Annu. Northeast Bioeng. IEEE Conf. Proc., pp. 119-120, 1996.

[6] J. Bronkorsta, P.J.H., Reinders, M.J.T., Hendriks, E.A., Grimbergen and G. Heethaar, R.M. \& Brakenhoff, "On-line detection of red blood cell shape using deformable templates," Pattern Recognit. Lett. 21, pp. 413- 424, 2000.

[7] J. P. and C. Neatpisarnvanit, "Red Blood Cell Extraction and Counting," 3rd Int. Symp. Biomed. Eng., 2008.

[8] H. A. Elsalamony, "Healthy and unhealthy red blood cell detection in human blood smears using neural networks," Micron, vol. 83, no. 4, pp. 32-41, 2016.

[9] N. V. K. Nishanth Rao, "Human Data Acquisition through Biometrics using LabVIEW," Int. J. Electr. Comput. Eng., vol. 7, no. 1, pp. 225-229, 2017.

[10] S. Madenda and S. Widodo, "Cursive Handwriting Segmentation Using Ideal Distance Approach," Int. J. Electr. Comput. Eng., vol. 7, no. 5, pp. 2863-2872, 2017.

[11] M. Hamiane and F. Saeed, "SVM classification of MRI brain images for computer-assisted diagnosis," Int. J. Electr. Comput. Eng., vol. 7, no. 5, pp. 2555-2564, 2017.

[12] B. Kanti Das, K. Kumar Jha, and H. Sekhar Dutta, "A new approach for segmentation and identification of disease affected blood cells,” Proc. - 2014 Int. Conf. Intell. Comput. Appl. ICICA 2014, pp. 208-212, 2014.

[13] T. Markiewicz and S. Osowski, "Data Mining Techniques for Feature Selection in Blood Cell Recognition," Eur. Symp. Artif. Neural Networks, no. April, pp. 26-28, 2006. 